\title{
Reliability Evaluation for the Surface to Air Missile Weapon Based on Cloud Model
}

\author{
Jianjun Deng ${ }^{1, a}$, Lin Zhang $^{1}$, Da Wu ${ }^{1}$ \\ ${ }^{1}$ Air and Missile Defense College, Air Force Engineering University in Xi'an, China
}

\begin{abstract}
The fuzziness and randomness is integrated by using digital characteristics, such as Expected value, Entropy and Hyper entropy. The cloud model adapted to reliability evaluation is put forward based on the concept of the surface to air missile weapon. The cloud scale of the qualitative evaluation is constructed, and the quantitative variable and the qualitative variable in the system reliability evaluation are corresponded. The practical calculation result shows that it is more effective to analyze the reliability of the surface to air missile weapon by this way. The practical calculation result also reflects the model expressed by cloud theory is more consistent with the human thinking style of uncertainty.
\end{abstract}

\section{Introduction}

The reliability is one of the important indexes of the surface to air missile weapon, it is an important part of tactical and technical indexes. The low index would affect operational use of missile weapon, the over high indexes are difficult to achieve, so equipment developing cost and cycle are increased, even lead to failure of developing work. The rational evaluation of reliability is an important problem of general demonstration for weapon system [1]. How to evaluate the reliability scientifically and effectively has become an important task of the reliability work. The conventional reliability evaluation uses an accurate count as reliability index regarding the parameter as an accurate count, which is inconsistent with the actual situation for the uncertainty of the reliability data. The evaluation is not accurate. The cloud theory is developing rapidly since it is put forward by Professor Deyi Li [2]. The transformation between qualitative concept and quantitative representation with cloud theory can overcome the theory defect caused by the results without fuzziness got by the fuzzy mathematics. It is more reasonable and effective to deal with the uncertain knowledge [3]. This paper analyses the reliability of surface to air missile weapon system with cloud model theory, which is used for describing the uncertain reliability data, evaluating the reliability of missile weapon system. The reliability index in the form of cloud model was got, realizing the transformation from the vague description to specific figures of the system reliability.

\section{The reliability evaluation of the Surface to air missile}

\footnotetext{
a Jianjun Deng: jj_dd_yy@163.com
}

The surface to air missile system consists of the Search radar, Track-guidance system, Lunch system and the Missile system [4], as shown in Figure1. The function of the four parts is supported respectively by the main technical indicators. Usually, reliability model of surface includes three kinds of model: the basic reliability model, the task reliability model and the combat reliability model. The basic reliability model is used to evaluate weapon system, parts fault and logistic support requirements, which is a series reliability model. Its basic information is derived by equipment that weapon performance required and equipment number of weapon composition. The task reliability model describes condition and degree of taskperforming. The combat reliability model is made up of combat readiness and combat work reliability. In order to show cloud model in reliability evaluation of surface to air missile system in the paper. The degree of missile weapon reliability is defined as follows, the probability of finish launching missile, hitting target and realizing the goal of battle mission in specified storage life , service period, specified application conditions and in specified time. The missile system is a complex system. Once a part of it breaks down, it is hard to determine its situation. So we should concerns more to what ex-tent the system can maintain the required functions.

Because of the complexity of the air missile weapon system, the fuzzy qualitative concepts method is usually more accurate than the precision method. On this occasion, the research for the transformation from qualitative concept to quantitative data becomes very important. This paper applies the cloud model to the comprehensive evaluation of the reliability of anti-air missile system. 


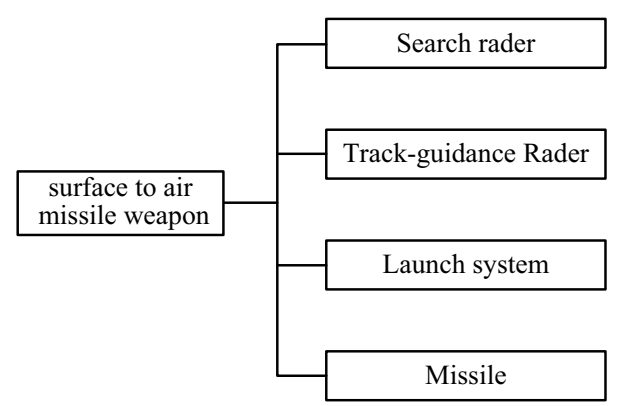

Figure 1. Composition block diagram of surface to air missile system

\section{Basic theory of the cloud model}

\subsection{Definition of the cloud}

Definition: Let $U=\{u\}$ as the discourse domain and $U$ is the common set, and $A$ is a fuzzy set in $U$. The membership degree of any element $u$ in $U$ to the fuzzy set $A, \mu_{A}(u)$ take the value in $[0,1]$ and the value is a stochastic number in a stability trend. A cloud is the mapping from the discourse domain $U$ to $[0,1]$. It is shown as follows,

$$
\mu_{A}(u): U \rightarrow[0,1] ; \forall_{u} \in U, u \rightarrow \mu_{A}(u)
$$

So the random distribution of $\mu_{A}(u)$ in $U$ forms a membership cloud. If the elements of discourse domain are simple and orderly, then $U$ is the basic variable, the degree of membership distribution in $U$ is defined as membership cloud. If the elements of discourse domain is not simple and orderly, then $U$ is mapped to other orderly discourse domain $U^{\prime}$ by a given rule $f$, one only one $u^{\prime}$ in $U^{\prime}$ corresponding to $u$ in $U$, so $U^{\prime}$ is the basic variable, the degree of membership distribution in $U^{\prime}$ is defined as membership cloud[5].

\subsection{The digital characteristics of cloud model}

Cloud model has three digital characteristics: Entropy $\left(E_{n}\right)$, Expected value $\left(E_{x}\right)$, and Hyper-Entropy $\left(H_{e}\right)$. The most extensive used cloud model is normal cloud model. The expect value $E_{x}$ is the most representative point of qualitative concept, the Entropy $E_{n}$ represents the measure of uncertainty, the greater value of the entropy is, the wider of the concept number range is, the fuzzier the concept is. The Hyper-Entropy $H_{e}$ is the uncertainty measure of $E_{n}$, which reflect the cohesion of uncertainty[5], the greater value of the Hyper-Entropy is, the greater value of the membership randomness is, the thicker the cloud is. Cloud model and its digital characteristics are shown in Figure2. It is shown that the fuzziness and randomness are integrated by three digital characteristics of cloud model, the mapping qualitative and quantitative is constituted.

\subsection{The Algorithm of cloud model}

The algorithm of forward normal cloud generator is shown as follows:
Input: the qualitative concept with Expected value $E_{x}$, Entropy $E_{n}$ and Hyper entropy $H_{e}$, and the number of cloud droplet is given.

Output: the quantitative location in number field of $N$ cloud droplet and degree of certainty represented for the concept of each cloud droplet.

Algorithm:

(1)Generating a normal random number with mean value $E_{n}$, standard deviation $H_{e}$, the number is $E_{n}{ }^{\prime}$;

(2)Generating a normal random number with mean value $E_{x}$, standard deviation $E_{n}$, the number is $x$;

(3)Let $x$ is a concrete quantization value of quantitative concept, it is cloud droplet;

(4) Computing $y=e^{\frac{-\left(x-E_{x}\right)^{2}}{2\left(E_{n}^{\prime}\right)^{2}}}$;

(5)Let $y$ is the membership degree of qualitative concept of $x$;

(6)The whole content of transformation between qualitative and quantitative is reflected by $\{x, y\}$;

(7) Step (1) (6) is repeated until $N$ cloud droplet are generated.

The algorithm can be calculated by MATLAB.

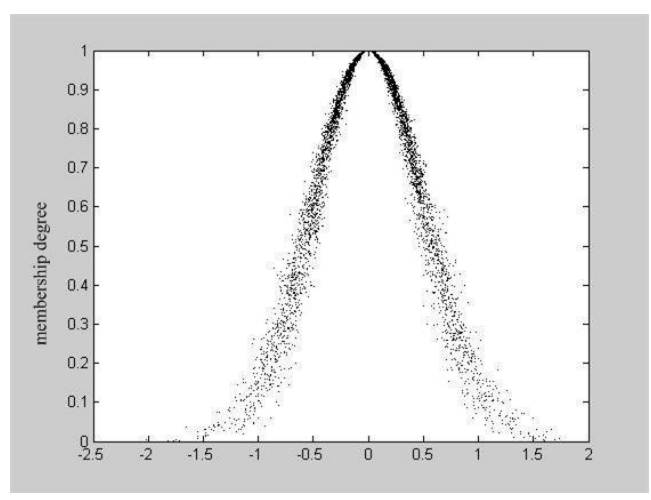

Figure 2. The Cloud model and its digital characteristics.

\section{Evaluation method of the cloud model}

\subsection{Get each index in Cloud model}

In the performance index system given in the system, the collected $n$ sample groups forms the decision matrix, then the $n$ exact numeric index can be represented by a cloud model. It is shown by Eq1 and Eq2.

$$
\begin{gathered}
E_{x}=\left(E_{x 1}+E_{x 2}+\ldots . E_{x n}\right) / n \\
E_{n}=\left(\begin{array}{l}
\max \left(E_{x 1}, E_{x 2}, \ldots E_{x n}\right)- \\
\min \left(E_{x 1}, E_{x 2}, \ldots E_{x n}\right)
\end{array}\right) / 6
\end{gathered}
$$

Meanwhile, each linguistic indicator can be represented by a cloud model, and then an index of $n$ language value can be represented by a one-dimensional cloud. It is shown by Eq3 and Eq4.

$$
\begin{aligned}
& E_{x}=\left(E_{x 1} E_{n 1}+E_{x 2} E_{n 2}+\ldots \ldots+E_{x n} E_{n n}\right) \\
& /\left(E_{x 1}+E_{x 2}+\ldots \ldots+E_{x n}\right)
\end{aligned}
$$




$$
E_{n}=\left(E_{n 1}+E_{n 2}+\ldots+E_{n n}\right)
$$

\subsection{Express the system station with many performance indexes with the cloud model}

Many of the system's performance indexes can be depicted by lots of cloud model, every index own its corresponding weight, so the system station can be expressed by the comprehensive cloud model. The comprehensive cloud model of two indexes considering corresponding weight can be got by the Eq5 and Eq6.

Definition: given the two cloud $C_{1}$ and $C_{2}$ on domain, described as the $C_{1}\left(E_{x l}, E_{n l}, H_{e l}\right), C_{2}\left(E_{x l}, E_{n l}, H_{e l}\right)$ respectively, and the other constant $\lambda$, the equation can be pushed out based on the arithmetic rule of cloud as follows[6]:

$$
\begin{gathered}
C_{1}+C_{2}=\left(E_{x 1}+E_{x 2}, \sqrt{E_{n 1}^{2}+E_{n 2}^{2}}, \sqrt{H_{e 1}^{2}+H_{e 2}^{2}}\right) \\
\lambda C=\left(\lambda E_{x}, \lambda E_{n}, \lambda H_{e}\right)
\end{gathered}
$$

In a similar way, multiple indexes comprehensive cloud model can be reasoned by the above equations.

\subsection{Remark set based on the evaluation of cloud model}

Use the remark set which consist of 11 remarks [7]:(extremely bad, worst, worse, bad, poor, normal, good, better, very good, best, extremely good) compose a evaluation cloud generator, as shown in Figure3.

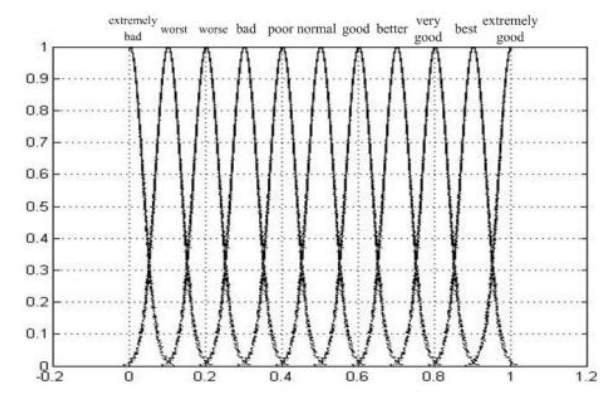

Figure 3. Evaluation cloud generator

\section{Application of the cloud model}

The reliability evaluation of surface to air missile system use expert evaluation method to evaluate the four parts' reliability. The result is shown in Table1

Table 1. Reliability evaluation of the surface to air missile.

\begin{tabular}{ccccc}
\hline & Search & Guidance & Launch & Missile \\
& system & system & system & system \\
\hline expert1 & normal & poor & better & better \\
expert 2 & good & normal & very good & good \\
expert 3 & very good & better & best & very good \\
& & & & \\
\hline
\end{tabular}

Through the cloud generator, the corresponding cloud model's $E_{x}$ of the experts evaluation result's linguistic values can serve as the quantitative express result, as shown in Table 2.

Table 2. Quantitative representation of the evaluation value.

\begin{tabular}{lcccc}
\hline & $\begin{array}{c}\text { Search } \\
\text { system }\end{array}$ & $\begin{array}{c}\text { Guidance } \\
\text { system }\end{array}$ & $\begin{array}{c}\text { Launch } \\
\text { system }\end{array}$ & $\begin{array}{c}\text { Missile } \\
\text { system }\end{array}$ \\
\hline expert 1 & 0.5 & 0.4 & 0.7 & 0.7 \\
expert 2 & 0.6 & 0.5 & 0.8 & 0.6 \\
expert 3 & 0.8 & 0.7 & 0.9 & 0.8 \\
\hline
\end{tabular}

The parameter of cloud model $E_{x} 、 E_{n}$ can be obtained by using Eq3 and Eq4, excess entropy $H_{e}$ should be adjust according to fuzzy extent of the remark, here take it 0.02 , as shown in Table3.

Table 3. Parameters of the subsystem reliability of cloud model.

\begin{tabular}{ccccc}
\hline & $\begin{array}{c}\text { Search } \\
\text { system }\end{array}$ & $\begin{array}{c}\text { Guidance } \\
\text { system }\end{array}$ & $\begin{array}{c}\text { Launch } \\
\text { system }\end{array}$ & $\begin{array}{c}\text { Missile } \\
\text { system }\end{array}$ \\
\hline$E_{x}$ & 0.63 & 0.53 & 0.8 & 0.7 \\
$E_{\mathrm{n}}$ & 0.1 & 0.1 & 0.1 & 0.1 \\
$\mathrm{He}$ & 0.02 & 0.02 & 0.02 & 0.02 \\
\hline
\end{tabular}

Through the analysis of surface to air missile weapon, we can get the weight of the reliability index of each system which is $(0.3,0.3,0.2,0.2)$. Take the weights into Eq5 to calculate, and then combine $E_{1}, E_{2}, E_{3}$ by Eq6, finally the cloud model $(0.665,0.06,0.012)$ of reliability evaluation was got.

Combine the evaluation result cloud figure and the assessment cloud figure, as shown in Figure 4. We determine the type of evaluation result cloud model according to its maximum certainty during the activation with assessment cloud model. We can infer from Figure 4 that the membership degree of evaluation result cloud model which has a "better" activation comment is obviously high, so the reliability evaluation result of this missile weapon system can be taken as "better".

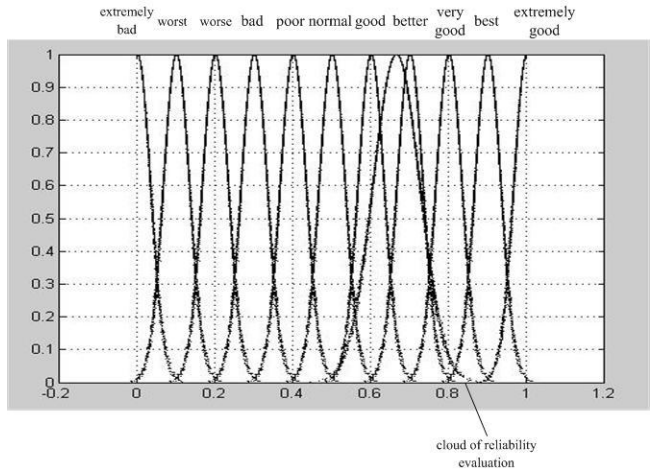

Figure 4. The cloud result of reliability evaluation

\section{Conclusions}

According to the cloud figures of reliability evaluation, the reliability evaluation based on the cloud model can be handled easily and used universally. The result is expressed by linguistic value, and is a distribution map showing the randomness and fuzziness of the reliability 
evaluation results [8]. The cloud model of reliability evaluation is more intuitive, more scientific and more in accordance with the human thinking mode.

\section{References}

1. N. Jin,S.Lou,J.Acad.Equip.Command.Techno, 17,61(2006)

2. D.Li, H.Meng, J .Computer R \& D, 32,16 (1995)

3. C.Liu, D.Li, Infor. Control, 34,236 (2005)

4. Y.Xin, W.Li, J. Syst. Eng. Electron, 25,316 (2003)

5. B.Xu, C.Wang, Chin. Astron. Astrophy,21,145 (2010)

6. Q.Li, J.Yul, Ship.Electron. Eng, 31,113 (2011)

7. J.Li, Y.Zhang, Computer.Digital.Eng, 38,58 (2010)

8. B.Liu, H.Li, Control. Decis, 24,957 (2009) 